\title{
Studies on molecular epidemiology of ESßL- producing Klebsiella pneumoniae isolated from patients hospitalized in a specialist hospital in southern Poland
}

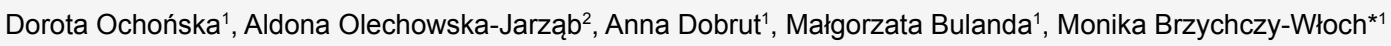

${ }^{1}$ Department of Molecular Medical Microbiology, Chair of Microbiology, Faculty of Medicine, Jagiellonian University Medical College, Krakow, Poland

Abstract $\quad{ }^{2}$ Center for Innovative Laboratory Diagnostics, John Paul II Hospital, Krakow, Poland

Introduction. Extended-spectrum $\beta$-lactamase (ESßL)-producing Klebsiella pneumoniae is currently one of the most common causes of nosocomial infections worldwide. The study aimed to characterize antibiotic resistance profile, the prevalence of selected genes encoding ES $\beta$ Ls, virulence, and the genetic relationship in $139 \mathrm{~K}$. pneumoniae isolates identified in John Paul II Specialist Hospital in Southern Poland, collected in 2016.

Materials/Methods. Bacterial identification and the preliminary antibiotic susceptibilities was performed using the VITEK $^{\circledR} 2$ Compact automated system. Genes encoding ES $\beta$ Ls were amplified by CTX-Mplex PCR and PCR reactions. The presence of nine genes encoding virulence factors was studied by multiplex PCR. Clonality was investigated by PFGE after digestion with Spel endonuclease.

Results. K. pneumoniae were mostly recovered from the respiratory tract $(40.3 \%)$, urine $(32.4 \%)$, wound swabs (19.4\%) and blood (5\%). In summary, $\mathbf{8 2 . 7 \%}$ of strains were classified as multidrug resistant (MDR). All isolates were confirmed as ES $\beta$ L producers and carried bla $a_{\text {CTX-M-type }}(85.6 \%)$, bla $a_{\text {SHV }}(82 \%), b / a_{\text {TEM }}(77.7 \%)$, bla CTX-M-9 $_{2}(75.6 \%)$ and bla $a_{\text {CTX-M-1 }}(1.4 \%)$ in various combinations. Moreover, triple bla genes were observed in $72 \%$ of isolates. The most common virulence-associated genes found among the isolates were entB (91.4\%), ybtS (55.4\%), iutA $(55.4 \%), \operatorname{magA}(53.2 \%), k f u(14.4 \%), \mathrm{K} 2$ $(11.5 \%)$, mrkD (10.1\%), rmpA $(7.9 \%)$ and allS (5\%). The PFGE analysis identified 4 major clusters (A-D) comprising $61 \%$ of the entire collection.

Conclusions. Our results indicate that the presence of a wide variety of MDR K. pneumoniae harbor ES $\beta$ Ls and virulence genes. Studies on molecular epidemiology of ES $\beta$ L-producing K. pneumoniae isolates are needed, particularly for epidemiological surveillance in the hospital environment.

Keywords

ES $\beta L$-producing K. pneumoniae $\cdot M D R \cdot$ molecular epidemiology $\cdot P F G E \cdot$ virulence-associated genes

Received: 02.12.2020, Accepted: 09.08.2021

\section{INTRODUCTION}

The effective worldwide spread of extended-spectrum $\beta$-lactamase (ES $\beta$ L)-producing Enterobacteriaceae, particularly Klebsiella pneumoniae, remains a critical concern for therapies against multidrug-resistant (MDR) bacteria [1]. K. pneumoniae is an opportunistic ESKAPE pathogen responsible for a wide spectrum of infections, including bacteremia, pneumonia, meningitis, purulent abscesses, urinary tract infections, and wound infections, at diverse sites [2]. Additionally, K. pneumoniae is known for its high prevalence and variety of antimicrobial resistance (AMR) genes [3]. These microorganisms are intrinsically resistant to ampicillin due to the presence of the SHV-1 penicillinase in their chromosomes. Resistance to additional drugs sporadically occurs through chromosomal mutations; however, most AMR in this species results from acquisition of these genes via HGT (horizontal gene transfer) mainly via large conjugative plasmids [3]. The most common mechanism of resistance to oxyimino-cephalosporins observed in $K$. pneumoniae is the production of ES $\beta$ Ls (extended-spectrum $\beta$-lactamases), followed by AmpC-like cephalosporinases, and KPC (Klebsiella pneumoniae carbapenemase) [4].

ES $\beta$ Ls are $\beta$-lactamases able of conferring bacterial resistance to the penicillins; first-, second-, third-generation cephalosporins; and aztreonam (but not the cephamycins or carbapenems) by hydrolysis of these antibiotics, which are inhibited by $\beta$-lactamase inhibitors such as clavulanic 
acid [5]. In recent years, most ES $\beta$ L-producing organisms have also been found to be resistant to other major classes of antibiotics, such as aminoglycosides, fluoroquinolones, and sulfonamides, owing to the coexistence of associated resistant genes encoded in and transmitted by plasmids [6].

$E S \beta L$-producing bacteria were first detected in Europe in the 1980s. Although the initial reports were from Germany and England, the vast majority of reports in the first decade after the discovery of ES $\beta$ Ls were from France [5]. According to the SENTRY Antimicrobial Surveillance Program (1997 to 2016), the prevalence rate of ES $\beta L$-producing Enterobacteriaceae in 4 main geographic areas is high, although the rates vary substantially in different regions: Europe (19.5\%), North America (12.3\%), Latin America (11\%), and Asia-Pacific (10.2\%) [7]. The study conducted in 16 European countries over 20 years reported that ES $\beta L$ screen-positive Enterobacteriaceae showed an increasing trend from $10.3 \%$ in $1997-2000$ to $24 \%$ in $2013-2016$, and the percentage of ES $\beta$ L screen-positive $K$. pneumoniae isolates increased from $7.1 \%$ to $19.4 \%$ [7]. In this global survey, MDR K. pneumoniae rates decreased from $18.9 \%$ in 2013 to $15.8 \%$ in 2016; however, in many European countries these rates ranged from $16.9 \%$ to $55.7 \%$ in 2016 [7]. Meanwhile, the proportions of MDR K. pneumoniae in the study by Chong et al. in 2018 were $15 \%-60 \%$ [1]. By contrast, already in 2016, Morris et al. published data from the European Antimicrobial Surveillance Network which indicated that across Europe the percentages of invasive $K$. pneumoniae resistant to thirdgeneration cephalosporins ranged from $0 \%$ to $70.1 \%$, with ES $\beta$ L producers accounting for between $85 \%$ and $100 \%$ of all strains reported [8].

The global problem of the presence of ES $\beta$ L-producing K. pneumoniae is treated with great care; but still, the epidemiological situation in individual countries varies. In Poland, it is diverse, as it ranges from sporadic cases to dangerous epidemic outbreaks. Due to the small number of scientific reports on the spread of pathogenic $K$. pneumoniae strains in southern Poland, there is a need to learn more about the epidemiological situation in highly specialized hospitals in this area. This need served as a launching pad for carrying out this study.

Our work aimed to characterize a collection the clinical isolates of $K$. pneumoniae with an emphasis on the antibiotic resistance profile, identification of ES $\beta L$ genes, virulenceassociated gene detection, and the genetic relationship, thereby helping guide the monitoring of the status, prevalence, and intra-hospital spread of such pathogenic strains.

\section{MATERIAL AND METHODS}

\section{Bacterial strains}

A total of $139 \mathrm{~K}$. pneumoniae isolates were collected from adult patients hospitalized at the John Paul II (JPII) Specialist Hospital in Krakow (Poland). The study period ran from January 1 to December 31, 2016. Most of the patients came from five different hospital wards: Cardiology and Cardiac Surgery with Cardiac Rehabilitation and Prevention (CARDIO); Pulmonology and Chest Medicine (PULMO); Neurology (NEURO); The Ward of Pediatric Infectious Diseases and Hepatology (INFECT DIS); and The Center for Diagnosis, Prevention and Telemedicine (DIAGNOST). K. pneumoniae isolates were mainly obtained from the respiratory tract $(40.3 \%, \mathrm{n}=56)$ and included: bronchial aspirates $(62.5 \%$, $\mathrm{n}=35)$; bronchoalveolar lavage, BAL $(16.1 \%, \mathrm{n}=9)$; sputum $(10.7 \%, n=6)$; microball $(3.6 \%, n=2)$; sore swabs $(3.6 \%, n=2)$; pleural fluid $(1.8 \%, n=1)$; and vac swab $(1.8 \%, n=1)$. Isolates were also derived from: urine $(32.4 \%, n=45)$; wound swabs $(19.4 \%, n=27)$; blood $(5 \%, n=7)$; fecal samples $(1.4 \%, n=2)$; catheter JJ $(0.8 \%, n=1)$; and urethral swab $(0.8 \%, n=1)$.

\section{Data collection}

The demographic information (sample type, ward) of the patients and ES $\beta$ L-producing isolate information (number, infectious site, and antibiotic susceptibility profile) were collected from the microbiological laboratory in the hospital.

\section{Species identification, antimicrobial susceptibility test- ing, and phenotype definitions}

The initial species identification, performed by the laboratory staff as part of routine diagnostics, was based on the assays of biochemical profiles of the isolates using the VITEK ${ }^{\circledR} 2$ Compact system with GN ID card (bioMérieux, France). In vitro susceptibility of $K$. pneumoniae to 15 antibacterial agents including penicillins (amoxicillin/clavulanic acid, piperacillin/ tazobactam), cephalosporins (ceftazidime, cefotaxime, cefepime), carbapenems (imipenem, meropenem), monobactams (aztreonam), aminoglycosides (amikacin, gentamicin), fluoroquinolones (ciprofloxacin, norfloxacin), and miscellaneous agents (colistin, nitrofurantoin, trimethoprim/ sulfamethoxazole) was analyzed by the VITEK ${ }^{\circledR} 2$ Compact system with AST-N332 cards according to the EUCAST recommendations and interpreted according to the EUCAST breakpoints [9]. The reference strain was Escherichia coli ATCC $\circledast 25922^{\mathrm{TM}}$. ES $\beta$ L production was confirmed by the standard double disc synergy test (DDST) [10]. Multidrugresistant (MDR) isolates were defined as non-susceptible to at least one agent in three or more antibiotic categories in accordance with the international protocol proposed by Magiorakos et al. [11]. 


\section{$E S \beta L$ gene identification}

Confirmed ES $\beta$ L-producing strains were screened for bla ${ }_{\mathrm{SHV}}$ and $b_{\text {TEM }}$ by polymerase chain reaction (PCR) assays using primers and protocols as described previously [12]. A multiplex PCR assay (CTX-Mplex PCR) was used to detect $b / a_{\text {CTX-M-type }}, b / a_{\text {CTX-M-1, }}$ and bla $a_{\text {CTX-M-9 }}$ genes, as described by Xu et al. [13].

\section{Virulence-associated gene detection}

Multiplex PCR assays as published by Compain et al. were used to detect the presence of nine virulence-associated genes, including those encoding for regulators of capsular serotype $\mathrm{K} 1$ and hypermucoviscosity phenotype (magA), capsular serotype K2 and hypermucoviscosity phenotype (K2), mucoid phenotype A (rmpA), type 3 adhesins (mrkD), allantoin metabolism (allS), iron transport and phosphotransferase function (kfu), enterobactin (entB), yersiniabactin (ybtS) and aerobactin siderophore system (iutA) [2].

\section{Pulsed field gel electrophoresis (PFGE)}

The genetic relatedness of the ES $\beta$ L-producing K. pneumoniae isolates was examined by pulsed field gel electrophoresis (PFGE) following the extraction of genomic DNA and digestion with Spel as the restriction enzyme and under the following experimental conditions: $0.5 \mathrm{~s}-20 \mathrm{~s}$ pulses for $18 \mathrm{~h}$, $14^{\circ} \mathrm{C}, 6 \mathrm{~V} / \mathrm{cm}^{2}$ [14]. K. pneumoniae ATCC $®$ BAA- $1705^{\mathrm{TM}}$ was used as the reference strain. The electrophoresis was run on the CHEF DR®II system (Bio-Rad Laboratories, CA, USA). Images were captured on the Gel Doc 2000 system (BioRad). The PFGE patterns were analyzed using GelCompar II and BioNumerics software packages (version 6.5, Applied Maths, Belgium). Similarity analysis was performed by Dice coefficients with customized tolerance of $1 \%$. Additionally, PFGE types and subtypes were discerned visually according to the criteria by Tenover et al. [15].

\section{Statistical analysis}

All statistical analyses were performed using $R$ (version 3.5.3; The R Foundation for Statistical Computing, Austria). Statistical significance was assessed via the Fisher's exact test for categorical variables in order to compare the resistance to selected antibiotics and the presence of virulence factors among the four main PFGE clonal types identified (A-D). The correlation analysis between virulence genes and resistance genes in the studied $K$. pneumoniae isolates was performed by calculating the Pearson's linear correlation coefficient $\left(r_{x y}\right)$. $P$-values of $<0.05$ were considered statistically significant.

\section{RESULTS}

\section{Antibiotic resistance patterns}

All examined K. pneumoniae $(100 \%, n=139)$ were resistant to amoxicillin with clavulanic acid, ceftazidime, cefotaxime, cefepime, and aztreonam. These isolates also showed different degrees of resistance to other antibiotics, such as norfloxacin (99.3\%, $n=138)$; ciprofloxacin (90.6\%, $n=126)$; trimethoprim/sulfamethoxazole (88.5\%, $n=123)$; piperacillin/ tazobactam (54.7\%, $n=76)$; gentamicin $(68.3 \%, n=42)$; and amikacin $(0.7 \%, n=1)$ [Fig. 1]. In summary, $82.7 \%(n=115)$ of the strains were classified as MDR.

\section{$E S \beta L S$}

The isolates were phenotypically confirmed as ES $\beta L$ producers and carried bla СTX-M-type $(85.6 \%, \mathrm{n}=112)$; followed by bla $_{\text {SHV }}(82 \%, n=114)$ and bla TEM $_{\text {M }}(77.7 \%, n=108)$. The strains produced mostly ES $\beta$ L-types CTX-M-9 (75.6\%, $n=105)$ and less frequently CTX-M-1 $(1.4 \%, n=2)$. Our analysis of the co-occurrence of the detected ES $\beta L$-encoding genes among the strains tested has revealed that the most common phenomenon is the co-existence of three genes observed in $72 \%(\mathrm{n}=100)$ of the isolates, including $b / a_{\mathrm{CTX}-\mathrm{M}-9} / b / a_{\mathrm{SHV}} / b / a_{\text {TEM }}$ $(65.5 \%, \quad \mathrm{n}=91) ; \quad b / a_{\text {СTX-M-type }} / b / a_{\text {SHV }} / b / a_{\text {TEM }}(5.8 \%, n=8)$ and $b l a_{\text {СTX-M-1 }} / b / a_{\text {SHV }} / b l a_{\text {TEM }}(0.7 \%, n=1)$. We have even observed that $5 \%(n=7)$ of strains harbored bla ${ }_{\text {СTX-M-9 }} / b / a_{\text {SHV }}, 2.9 \%(n=4)$ of strains harbored $b / a_{\text {CTX-M-9 }} / b / a_{\text {TEM }}, 2.2 \%(n=3)$ of strains harbored $b / a_{\mathrm{SHV}} / b / a_{\mathrm{TEM}}$, and $1.4 \%(\mathrm{n}=2)$ of strains harbored bla $_{\text {CTX-M-type }} / b_{\text {ShV }}$. Nevertheless, considering $6.5 \%(n=9)$ of isolates having only a single $\beta$-lactamase gene, we noted a predominance of bla ${ }_{\text {СТХ-M-9 }}(2.2 \%, n=3)$.

\section{Virulence}

Multiplex polymerase chain reaction analysis demonstrated that the kfu and mrkD genes, encoding iron transport and phosphotransferase function and type 3 fimbrial adhesins, were present in $14.4 \%(n=20)$ and $10.1 \%(n=14)$ of isolates, respectively. The regulators of the mucoid phenotype $A$ $(r m p A)$ gene were detected in $7.9 \%(n=11)$ of isolates. Seven isolates $(5 \%)$ were positive for allS gene encoding allantoin metabolism. Additionally, the enterobactin (entB) gene was found in $91.4 \%(n=127)$, the yersiniabactin ( $y b t S)$ gene in $55.4 \%(n=77)$, and the aerobactin siderophore system (iutA) gene in $55.4 \%(n=77)$ of isolates. Seventy-four isolates (53.2\%) presented the capsular serotype K1 (magA gene), and the capsular K2 (K2 gene) was found in $11.5 \%(n=16)$ of isolates. Thirty-nine combinations of genes encoding selected virulence factors were observed, with clear dominance of: $y b t S / e n t \mathrm{~B} /$ iutA in $16.5 \%(\mathrm{n}=23)$ of isolates, $y b t \mathrm{~S} / \mathrm{ent} \mathrm{B} / \mathrm{magA}$ in $14.4 \%(n=20)$, and $y b t S / e n t B / i u t A / m a g A$ in $8.6 \%(n=12)$. 


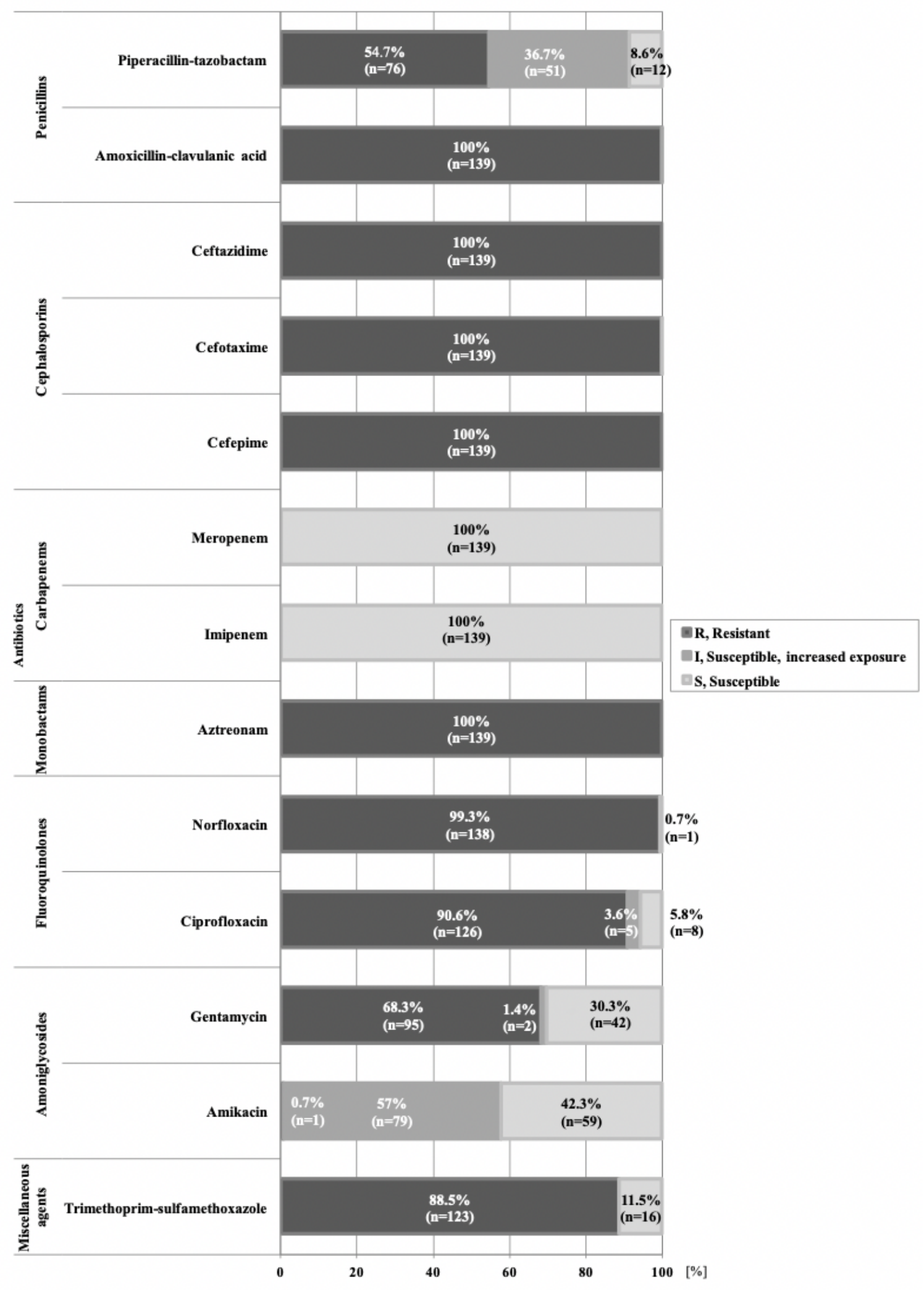

Figure 1: Antimicrobial resistance patterns of ESßL-producing K. pneumoniae isolates. 


\section{Correlation between virulence genes and resistance genes}

Twenty-five different combinations were observed based on the coexistence of virulence genes with resistance genes [Fig. 3]. The largest group of isolates $(n=52,37.4 \%)$ were strains with three genes encoding virulence factors and four resistance genes in different lists: $b / a_{\mathrm{SHV}} / \mathrm{bla}_{\mathrm{TEM}} / \mathrm{bla} \mathrm{a}_{\mathrm{CTX}-\mathrm{M}-\mathrm{type}} /$ bla CTX-M- $9+y b t S / e n t B / i u t A(n=17,32.7 \%) ; b_{\text {SHV }} / b l a_{\text {TEM }} /$ bla $a_{\text {CTX-M-type }}$ bla $a_{\text {CTX-M-9 }}+y b t S / e n t B / m a g A \quad(n=51,28.8 \%) ; b l a_{\mathrm{SHV}} / b l a_{\mathrm{TEM}} / b l a_{\text {СTX }}$ ${ }_{\text {M-yype }} /$ bla $a_{\text {СтХ-м-9 }}+$ entB/iutA/magA $(\mathrm{n}=10,19.2 \%) ; \quad b / a_{\text {SHV }} / b^{\prime} / a_{\text {TEM }} /$ bla $_{\text {CTX-M-type }} /$ bla $a_{\text {CTX-M-9 }}+$ entB/iutA/magA $\quad(\mathrm{n}=10, \quad 19.2 \%) ; \quad b l a_{\text {SHV }} /$ $b / a_{\text {TEM }} / b l a_{\text {CTX-M-type }} / b l a_{\text {CTX-M-9 }}+m r k D /$ entB/iutA $(\mathrm{n}=6,11.5 \%) ; b l a_{\text {SHV }} /$

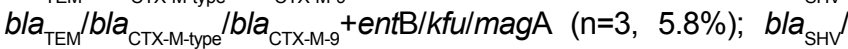
$b / a_{\text {TEM }} / b l a_{\text {CTX-M-type }} / b l a_{\text {CTX-M-9 }}+y b t S /$ entB/magA $(n=1,1.9 \%)$. The obtained Pearson's coefficient $\left(r_{x y}=0.27, P=0.001\right)$ indicates a weak correlation $\left(0.1 \leq r_{x y}<0.3\right)$ between the coexistence of virulence genes and resistance genes in the studied $K$. pneumoniae isolates.

\section{PFGE}

PFGE identified 41 different profiles which fell into 4 major clusters (A-D) in 85 isolates (comprising $61 \%$ of the entire collection), based on a similarity of $\geq 98 \%$ (cut-off) [Fig. $2]$. The largest cluster, $A$, accounted for $28.1 \% \quad(n=39)$ of all isolates and contained strains from four hospital wards, including $19(48.7 \%)$ isolates from CARDIO, $15(38.5 \%)$ from PULMO, 4 (10.2\%) isolates from NEURO, and 1 (2.6\%) isolate from the DIAGNOST ward. The next clusters were $\mathrm{B}(16.5 \%, \mathrm{n}=23)$ with $14(60.9 \%)$ isolates from CARDIO, 6 (26\%) isolates from PULMO, 2 (8.7\%) isolates from NEURO, and $1(4.3 \%)$ isolate from the DIAGNOST ward; cluster C $(8.6 \%, n=12)$ with $7(58.3 \%)$ isolates from CARDIO, $3(25 \%)$ isolates from PULMO, 1 (8.3\%) isolate from INFECT DIS, and $1(8.3 \%)$ isolate from the DIAGNOST ward; cluster D $(7.2 \%, n=10)$ with $7(70 \%)$ isolates from CARDIO, $2(50 \%)$ isolates from PULMO, and $1(10 \%)$ isolates from INFECT DIS. Clone A was mostly isolates extracted from bronchial aspirates (46\%), clones B and C were mostly isolates from urine $(34.8 \%$ and $41.7 \%$, respectively), clone D was mostly isolates from urine (50\%). Isolates classified into clonal type A demonstrated a significantly higher sensitivity to trimethoprim/sulfamethoxazole than isolates from the other three main PFGE clonal types (B-D) $(p=0.06)$. Isolates classified into clonal type $\mathrm{C}$ demonstrated a significantly lower resistance to amikacin and piperacillin/tazobactam than isolates from the remaining main clonal types $A, B, D$ $(p<0.001)$. Isolates classified into clonal type $D$ showed a significantly higher sensitivity to gentamicin than isolates from the remaining clonal types $A, B$, and $C(p<0.001)$. As for other antibiotics, the results were not statistically significant. Among the main clonal types $A-D$, three subgroups were distinguished, which differed as regards the presence of bla $_{\text {SHV }}$ statistically significantly $(p<0.001)$, starting from the highest detectability: D (93.75\%), A (82.05\%), B and C ( $79.17 \%$ and $77.78 \%$, respectively). No statistically significant differences were found between the affiliation of the strains to main clonal types (A-D) and virulence, the type of the ward, or the kind of clinical material they were isolated from. The remaining strains, $16 \%(n=22)$, were clustered into 7 minor clonal lineages: $G(n=3), H(n=3), I(n=2), J(n=2), K(n=2), L$ $(n=2), M(n=2)$, and 32 singletons.

\section{DISCUSSION}

ES $\beta$-producing Enterobacteriaceae are among the most common pathogens involved in nosocomial infections worldwide, with species other than Escherichia coli (especially $K$. pneumoniae) being increasingly reported in recent years [16].

In K. pneumoniae rods, ES $\beta$ Ls are still some of the most important mechanisms of drug resistance clinically and epidemiologically, due to their potential for rapid spread, as the genes encoding them are usually transferred on mobile genetic elements such as plasmids. Moreover, the widespread use of antibiotics has led to the emergence of MDR strains which accumulate mechanisms conditioning resistance to various groups of antibiotics. Currently, such strains are a global problem $[3,6,7,17]$. Studies on molecular epidemiology of ES $\beta$-producing $K$. pneumoniae isolates are needed, particularly under multi-level epidemiological monitoring, which is considered essential to understanding trans-hierarchical evolutionary changes in antibiotic resistant populations, and ultimately to establish appropriate control interventions [16].

This study investigates drug resistance profiles, the presence of genes encoding selected virulence factors, and genetic diversity of the ES $\beta$ L-producing $K$. pneumoniae strain collection. Considering the latest research, this examination is part of a general trend of research on the molecular epidemiology of the species $K$. pneumoniae, and the obtained strain characterization results are similar to the results presented by other Polish authors [4, 18, 19, 20].

In their analysis of hospital-acquired infections caused by ES $\beta$ L-producing $K$. pneumoniae, Castanheira et al. demonstrated that these rods were mainly the cause of wound infections $(20.8 \%)$, respiratory tract infections $(16.3 \%)$, urinary tract infections (13\%), and generalized infections (11.9\%) [7]. Therefore, respiratory secretions, urine, wound swabs, and blood are the main materials from which these pathogens were isolated [7]. In our study, the greatest number of $K$. pneumoniae isolates $(40.3 \%)$ came from materials from the respiratory tract, urine $(32.4 \%)$, wound swabs $(19.4 \%)$, and blood (5\%). Similarly, Wang et al. described 


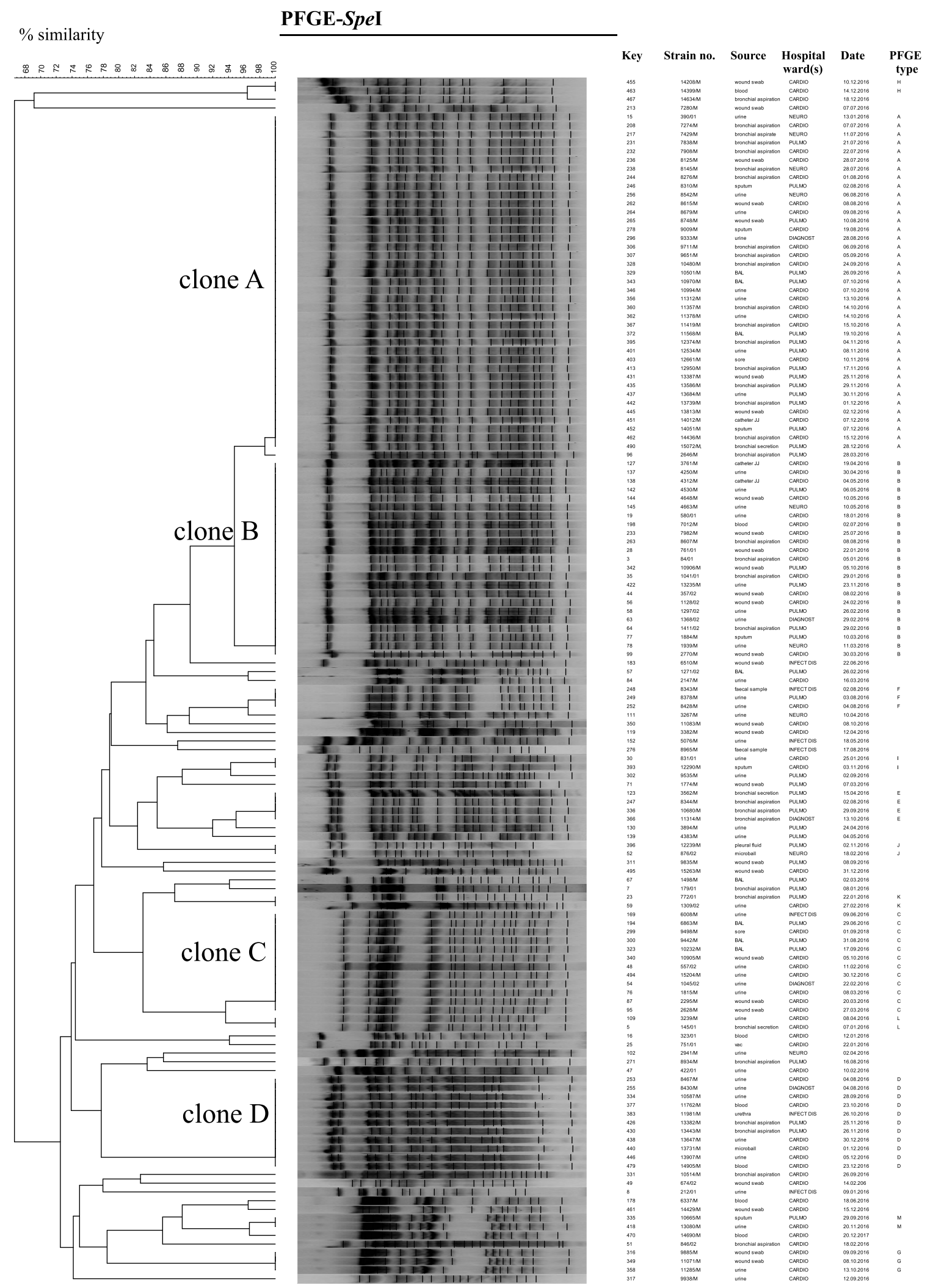

Figure 2. Dendrogram illustrating the PFGE patterns of $139 \mathrm{ES} \beta \mathrm{L}$-producing $K$. pneumoniae isolates after restriction with Spel enzyme. PFGE settings: Similarity coefficient: Dice, Optimization: $1 \%$, Tolerance: $1 \%$, Clustering method: UPGMA. 


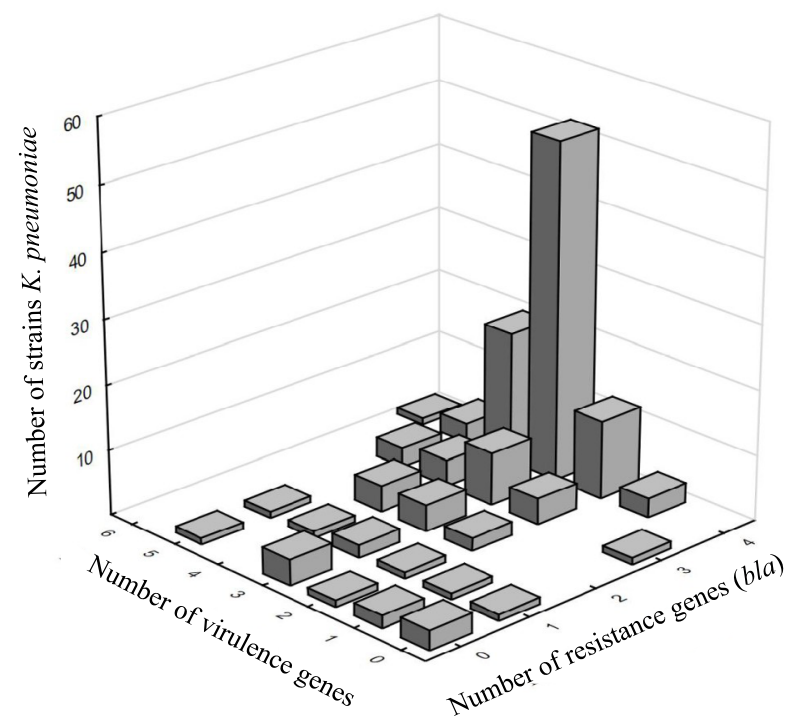

Figure 3. Correlation between virulence genes and resistance genes in ES $\beta$ L-producing K. pneumoniae isolates. Pearson's correlation coefficient $r_{x y}=0.27, P=0.001$.

most $K$. pneumoniae isolates that were cultured from urine $(38.8 \%)$, materials from the respiratory tract $(28.25 \%)$, followed by wound swabs (11.04\%) and blood (7.22\%) [6]. Correspondingly, most $K$. pneumoniae isolates characterized by Ranjbar et al. came from urine $(63.75 \%)$, blood $(37.5 \%)$, sputum (35.21\%) and wounds (33.33\%) [21].

The results of drug susceptibility testing as regards the $K$. pneumoniae collection under study demonstrated a resistance profile to $\beta$-lactam antibiotics that is typical of ES $\beta L$ producers. All isolates were found to be resistant to several marker oxyimino- $\beta$-lactams of the ES $\beta L$ mechanism: for instance, ceftazidime, cefotaxime, cefepime and aztreonam.

It should be underlined that our investigations revealed a high percentage (54.7\%) of strains resistant to piperacillin/ tazobactam. Similar results were obtained by other Polish researchers, Mrowiec et al., who recorded $53.5 \%$ of $K$. pneumoniae isolates resistant to this antibiotic in the adult population [18]. On the other hand, an investigation carried out by Korean researchers, Lee et al., found a lower percentage $(20.9 \%)$ of $K$. pneumoniae strains which were resistant to piperacillin/tazobactam [22]. In his 1995 study, Livermoore proved that ES $\beta$ L-producing strains frequently turn out to be resistant in vitro to combinations of $\beta$-lactams with inhibitors, despite the fact that ES $\beta L s$, by definition, are inhibited by $\beta$-lactamase inhibitors (clavulanic acid, sulbactam, or tazobactam) [23]. Furthermore, literature data indicate that the development of resistance to piperacillin/ tazobactam in clinical $K$. pneumoniae isolates may be the result of several different resistance mechanisms, including the hyperproduction of constitutive chromosomal $\beta$-lactamases SHV-1 or the hyperproduction of TEM-1 $\beta$-lactamase due to the presence of either strong promoters or multiple bla TEM-1 $_{1}$ copies [22]. In our study, among all isolates resistant to piperacillin/tazobactam, as many as $93.4 \%$ of the strains demonstrated the presence of several (2-3) variants of ES $\beta L$ enzymes. Hence, the observed growing resistance to piperacillin/tazobactam in $K$. pneumoniae is caused by the co-occurrence of different variants of ES $\beta L$ enzymes in individual strains.

In this study, examinations conducted using PCR confirmed the presence of genes encoding ES $\beta L$ in all the studied K. pneumoniae isolates; in as many as $72 \%$ of the strains, genes belonging to three different families were detected: CTX-M-type (85.6\%), SHV (82\%), and TEM (77.7\%). Within the cefotaxime group, however, a clear dominance of the CTX-M-9 enzyme group was demonstrated at $75.6 \%$, while the presence of CTX-M-1 was confirmed in only two $(1.4 \%)$ strains. The study showed a high percentage of isolates $(65.5 \%)$ that were characterized by the coexistence of three genes: $b / a_{\text {СTX-M-9 }} / b / a_{\text {SHV }} / b / a_{\text {TEM }}$. We have even observed that $5 \%$ of strains harbored $b / a_{\text {СTX-M-9 }}$ and $b / a_{\mathrm{SHV}} 2.9 \%$ of strains harbored bla ${ }_{\mathrm{CTX-M-9}}$ and bla $a_{\mathrm{TEM},}$ and $2.2 \%$ of strains harbored bla $_{\mathrm{SHV}}$ and bla $\mathrm{TEM}_{\mathrm{TE}}$. Contrarily, in 2019, a similar Polish research into $K$. pneumoniae isolates carried out by Mrowiec et al. showed that $91.2 \%$ of strains carried bla CTX-M-1 $_{1}, 63.5 \%$ carried $b / a_{\mathrm{TEM}}$ and $11.8 \%$ carried $b / a_{\mathrm{SHV}} ; 7 \%$ of strains had all 3 genes, $67.4 \%$ had $b / a_{\text {CTX-M-1 }}$ and $b / a_{\text {TEM }}, 1.2 \%$ had $b / a_{\text {TEM }}$ and $b l a_{\mathrm{SHV}}$ and $1.2 \%$ had bla $\mathrm{CTX}_{\mathrm{CT}-1}$ and bla ${ }_{\mathrm{SHV}}$ [18]. However, the $K$. pneumoniae isolates coming from 2011 and described by Walter de Walthoffen et al. in Poland demonstrated the presence of $b / a_{\text {СTX-M }}$ at $94 \%, b l a_{\text {TEM }}$ at $94 \%$, and $b l a_{\mathrm{SHV}}$ at $52.8 \%$, while at the same time, $41.6 \%$ of strains had all 3 genes, $86.1 \%$ had $b / a_{\text {TEM }}$ and $b / a_{\text {СTX-M }}, 50 \%$ had $b / a_{\text {SHV }}$ and bla ${ }_{\text {CTX-M, }}$ and $44.4 \%$ had $b / a_{\text {TEM }}$ and bla $a_{\mathrm{SHV}}$ [24]. The studies quoted above show that a spread of many different variants of ES $\beta$ L enzymes is observed in Poland. In Portugal, studies carried out by Rodrigues et al. confirm the fact that, among $K$. pneumoniae strains, there is coexistence of $\beta$-lactamases belonging to CTX-M-15, SHV and TEM, detected at $47 \%$, $45 \%$, and $9 \%$, respectively [16]. Yet, as early as in 2003 in North America, Paterson et al. documented the occurrence of $K$. pneumoniae with multiple ES $\beta L$ types in different regions of the world, among others, Argentina, Australia, South Africa, Turkey, and United States [25].

A review of the literature indicates that in $K$. pneumoniae genes encoding plasmid-transmitted ES $\beta L \quad \beta$-lactamases often coexist with other plasmid-encoded resistance mechanisms, including to fluoroquinolones, aminoglycosides, co-trimoxazole, tetracyclines, and chloramphenicol [26]. The characterization of the collection of ES $\beta L$-producing $K$. pneumoniae strains that was carried out confirmed the high percentage of isolates resistant to antibiotics from therapeutic 
groups other than penicillins, such as fluoroquinolones (ciprofloxacin, 90.6\%), aminoglycosides (amikacin, 0.7\%) and trimethoprim/sulfamethoxazole (88.5\%). Studies carried out in Poland by Ojdana et al. found a high percentage of $K$. pneumoniae strains resistant to trimethoprim/ sulfamethoxazole (100\%), amikacin (58.3\%), and $16.7 \%$ of isolates resistant to ciprofloxacin [19]. In Brazil, Wang et al. recorded $10.5 \%, 59.8 \%$, and $80.6 \%$ of strains resistant to, respectively, amikacin, ciprofloxacin, and trimethoprim/ sulfamethoxazole [6]. The differences observed in the abovementioned investigations prove the fact that drug resistance profiles in individual ES $\beta$ L-producing $K$. pneumoniae strains vary and can be characteristic of a given region, hospital, or unit. In this study, $82.7 \%$ of the examined $K$. pneumoniae isolates were classified into the category of MDR strains, which is similar to the results of the Brazilian researchers, who recorded $84 \%$ of strains from this species with the MDR phenotype [26].

In the course of analysis of the most common genes encoding virulence factors in the studied pool of $K$. pneumoniae isolates, $55.4 \%, 10.1 \%$, and $7.9 \%$ demonstrated the presence of genes iutA, $m r k \mathrm{D}$, and $r m p \mathrm{~A}$, respectively. Contrarily, in the examination by Compain et al., the mrkD gene was detected in $100 \%$ of $K$. pneumoniae isolates, while the presence of the iutA gene was not found [2]. Also Ranjbar et al. demonstrated the presence of the $m r k D$ gene in $84.34 \%$ and the rmpA gene in $13.91 \%$ of the studied $K$. pneumoniae isolates [21]. On the other hand, Ferreira et al. recorded iutA in $40 \%$ and $m r k D$ in $96 \%$ of isolates, but they did not detect the presence of the rmpA gene [26]. Compain et al. showed the presence of the rmpA gene in $7.1 \%$ of isolates, which is similar to the proportion in this study (7.9\%). The remaining virulence-associated genes found among the isolates were entB (91.4\%), ybtS (55.4\%), kfu (14.4\%), K2 (11.5\%), allS (5\%). The French researchers also detected these virulence genes: entB (100\%), kfu (35.7\%), ybtS (21.4\%), and allS (7.1\%) [2], and the researchers from Brazil, respectively: entB (100\%), and ybtS (60\%) [26]. The magA gene was detected in $53.2 \%$ of the strains, contrary to Brisse et al. in France and Lascols et al. in Canada, who did not demonstrate the presence of the magA gene in any of the $K$. pneumoniae isolates studied $[27,28]$.

As for the K. pneumoniae strains under study, it was observed according to the data in the literature $[8,20]$, that the majority of the isolates $(61 \%)$ were of clonal origin. The assessment of the genetic similarity of the studied $K$. pneumoniae rods was done with the use of PFGE. According to Paterson and Bonomo, PFGE is the most frequently used molecular reference method to genotype ES $\beta L$-producing Enterobacteriaceae in the world [5]. It is confirmed by numerous international, as well as Polish, publications concerning molecular analysis of the $K$. pneumoniae population: in France, Ireland, Brazil, China, and Poland $[8,14,20,26,27]$. Our work showed good typeability, reproducibility of Spel for K. pneumoniae subtyping, and revealed high genetic diversity among the studied isolates. Analysis of the Spel-PFGE genetic profiles of the strains demonstrated that their genetic similarity ranged from $66.6 \%$ to $100 \%$. The highest degree of similarity $(100 \%)$ was found between isolates classified into four PFGE clonal types (A-D) and seven minor clonal lineages (G-M). These isolates came from various clinical materials from patients hospitalized in different hospital wards. The obtained results of genotyping indicate that $K$. pneumoniae epidemic clones spread between different hospital departments. Similarly, Morris et al. demonstrated clonal organization of the $K$. pneumoniae strain collection: among 137 isolates, they identified 111 various PFGE profiles, which fell into 16 major clusters (A-P) in 112 isolates based on similarity of $>85 \%$, with each cluster comprising 2-43 isolates [8]. The clonal genetic structure in $K$. pneumoniae was also confirmed by Lascols et al. in their work published in 2013, which identified 3 major clonal groups among the isolates under study with more than 10 isolates per cluster, 10 minor clusters with less than 10 isolates per cluster, and 13 singletons [28]. In our study, most isolates $(28 \%)$ were classified into clonal group A (cluster A), and in the research by Morris et al., the percentage of isolates classified into the largest clonal group (cluster A) was very similar and amounted to $31 \%$ (Morris et al. 2016). Yet, Lascols et al. described the largest clonal group (cluster C) comprising $53 \%$ of $K$. pneumoniae isolates [28].

The epidemiological situation of a hospital or a given ward may be influenced by many factors; among them, the specificity of the facility, the profile of patients that are treated there, the antibiotics employed, hygienic and sanitary measures undertaken or the persistence of certain pathogenic microorganisms.

To conclude, due to worldwide reports of a significant increase in resistance of $K$. pneumoniae to the antibacterial drugs used and the observed regional changes in the scale of this phenomenon, it is necessary to find the ES $\beta L+$ phenotype in all $K$. pneumoniae isolates as part of routine microbiological diagnostics. In addition, it should be remembered that when choosing empirical therapy for serious infections with an etiology of $K$. pneumoniae ES $\beta \mathrm{L}+$, it is extremely important to take into account the local epidemiological characteristics. The application of molecular techniques to identify multidrugresistant strains can contribute to improved surveillance and rapid detection of epidemic clones, and as a consequence, to a reduction in their spread in the hospital environment. 


\section{Acknowledgments}

We would like to thank the staff and employees of the microbiological laboratory at John Paul II Hospital in Krakow for their participation in the study. A special thank you to Catherine Gąsior-Kulasiak for English language editing.

\section{Authors' Contribution}

D.O.: research concept and design; supervising the project; carrying out the experiments; data analysis and interpretation; writing - original draft preparation; writing - review and editing; visualization; literature review; final proofreading and approval of the version for publication; funding acquisition; A.O.J.: acquisition of data; final proofreading and approval of the version for publication; A.D.: carrying out the experiments; final proofreading and approval of the version for publication; M.B.: acquisition of data; final proofreading and approval of the version for publication; M.B.W.: research concept and design; content-related supervision over research; final proofreading and approval of the version for publication.

\section{Funding}

This research was financially supported by the Jagiellonian University Medical College (Grant/K/ZDS/007829).

\section{Conflict of Interest}

The authors have no potential conflicts of interest to declare.

\section{Ethics Approval}

The study was approved by the Bioethics Committee of the Jagiellonian University in Krakow, Poland (KBET/1072.6120.264.2019).

\section{References}

[1] Chong Y., Shimoda S., Shimono N. (2018). Current epidemiology, genetic evolution and clinical impact of extended-spectrum ß-lactamase-producing Escherichia coli and Klebsiella pneumoniae. Infect. Genet. Evol., 61: 185-188.

[2] Compain F., Babosan A., Brisse S., Genel N., Audo J., Ailloud F., Kassis-Chikhani N., Arlet G., Decré D. (2014). Multiplex PCR for detection of seven virulence factors and K1/K2 capsular serotypes of Klebsiella pneumoniae. J. Clin. Microbiol., 52: 4377-4380.
[3] Wyres K.L., Holt K.E. (2018). Klebsiella pneumoniae as a key trafficker of drug resistance genes from environmental to clinically important bacteria. Curr. Opin. Microbiol., 45: 131-139.

[4] Baraniak A., Izdebski R., Fiett J., Sadowy E., Adler A., Kazma M., Salomon J., Lawrence C., Rossini A., Salvia A., et al. (2013). Comparative population analysis of Klebsiella pneumoniae strains with extended-spectrum $\beta$-lactamases colonizing patients in rehabilitation centers in four countries. Antimicrob. Agents Chemother., 57: 1992-1997.

[5] Paterson D.L., Bonomo R.A. (2005). Clinical update extendedspectrum $\beta$-lactamases : A clinical update. Clin. Microbiol. Rev., 18: 657-686.

[6] Wang Y., Zhang Q., Jin Y., Jin X., Yu J., Wang K. (2019). Epidemiology and antimicrobial susceptibility profiles of extendedspectrum beta-lactamase-producing Klebsiella pneumoniae and Escherichia coli in China. Braz. J. Microbiol., 50: 669-675.

[7] Castanheira M., Deshpande L.M., Mendes R.E., Canton R., Sader H.S., Jones R.N. (2019). Variations in the occurrence of resistance phenotypes and carbapenemase genes among Enterobacteriaceae isolates in 20 years of the SENTRY antimicrobial surveillance program. Open Forum Infect. Dis., 6: S23-S33.

[8] Morris D., O'Connor M., Izdebski R., Corcoran M., Ludden C.E., McGrath E., Buckley V., Cryan B., Gniadkowski M., Cormican M. (2016). Dissemination of clonally related multidrug-resistant Klebsiella pneumoniae in Ireland. Epidemiol. Infect., 144: 443-448.

[9] The European Committee on Antimicrobial Susceptibility Testing: Breakpoint tables for interpretation of MICs and zone diameters. Version 9.0, 2019. http://www.eucast.org

[10] CLSI: Performance Standards for Antimicrobial Susceptibility Testing. (2018). 28th ed. CLSI supplement M100. Wayne PA: Clinical and Laboratory Standards Institute.

[11] Magiorakos A., Srinivasan A., Carey R.B., Carmeli Y., Falagas M.E., Giske C.G., Harbarth S., Hindler J.F., Kahlmeter G., OlssonLiljequist B., et al. (2012). Multidrug-resistant, extensively drugresistant and pandrug-resistant bacteria: An international expert proposal for interim standard definitions for acquired resistance. Clin. Microbiol. Infect., 18: 268-281.

[12] Latifpour M., Gholipour A., Damavandi M.S. (2016). Prevalence of extended-spectrum beta-lactamase-producing Klebsiella pneumoniae isolates in nosocomial and community-acquired urinary tract infections. Jundishapur J. Microbiol., 9: e31179.

[13] Xu L., Ensor V., Gossain S., Nye K., Hawkey P. (2005). Rapid and

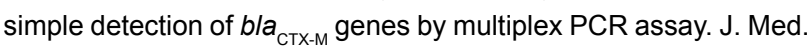
Microbiol., 54: 1183-1187.

[14] Han H., Zhou H., Li H., Gao Y., Lu Z., Hu K., Xu B. (2013). Optimization of pulse-field gel electrophoresis for subtyping of Klebsiella pneumoniae. Int. J. Environ. Res. Public Health, 10: 2720-2731.

[15] Tenover F.C., Arbeit R.D., Goering R.V., Mickelsen P.A., Murray B.E., Persing D.H., Swaminathan B. (1995). Interpreting chromosomal DNA restriction patterns produced by pulsed- field gel electrophoresis: Criteria for bacterial strain typing. J. Clin. Microbiol., 33: 2233-2239. 
[16] Rodrigues C., Machado E., Ramos H., Peixe L., Novais Â. (2014). Expansion of ESBL-producing Klebsiella pneumoniae in hospitalized patients: A successful story of international clones (ST15, ST147, ST336) and epidemic plasmids (IncR, IncFII $)$. Int. J. Med. Microbiol., 304: 1100-1108.

[17] Ny S., Kozlov R., Dumpis U., Edquist P., Gröndahl-Yli-Hannuksela K., Kling A.M., Lis D.O., Lübbert C., Pomorska-Wesołowska M., Palagin I., et al. (2018). Large variation in ESBL-producing Escherichia coli carriers in six European countries including Russia. Eur. J. Clin. Microbiol. Infect. Dis., 37: 2347-2354.

[18] Mrowiec P., Klesiewicz K., Małek M., Skiba-Kurek I., Sowa-Sierant I., Skałkowska M., Budak A., Karczewska E. (2019). Antimicrobial susceptibility and prevalence of extended-spectrum beta-lactamases in clinical strains of Klebsiella pneumoniae isolated from pediatric and adult patients of two Polish hospitals. New Microbiol., 42: 197-204.

[19] Ojdana D., Sacha P., Wieczorek P., Czaban S., Michalska A., Jaworowska J., Jurczak A., Poniatowski B., Tryniszewska E. (2014). The occurrence of $b / a_{\text {CTX-M }}, b l a_{\text {SHV }}$ and $b l a_{\text {TEM }}$ genes in extendedspectrum $\beta$-lactamase-positive strains of Klebsiella pneumoniae, Escherichia coli, and Proteus mirabilis in Poland. Int. J. Antibiot., 2014: 935842.

[20] Sękowska A., Gospodarek E., Kamińska D. (2012). Antimicrobial susceptibility and genetic similarity of ESBL-positive Klebsiella pneumoniae strains. Arch. Med. Sci., 8: 993-997.

[21] Ranjbar R., Kelishadrokhi A.F., Chehelgerdi M. (2019). Molecular characterization, serotypes and phenotypic and genotypic evaluation of antibiotic resistance of the Klebsiella pneumoniae strains isolated from different types of hospital-acquired infections. Infect. Drug Resist., 12: 603-611.

[22] Lee J., Oh C.E., Choi E.H., Lee H.J. (2013). The impact of the increased use of piperacillin/tazobactam on the selection of anti- biotic resistance among invasive Escherichia coli and Klebsiella pneumoniae isolates. Int. J. Infect. Dis., 17: e638-e643.

[23] Livermoore D.M. (1995). Beta-Lactamases in laboratory and clinical resistance. Clin. Microbiol. Rev., 8: 557-584.

[24] de Walthoffen S.W., Mlynarczyk A., Sawicka-Grzelak A., Durlik M., Paczek L., Chmura A., Ciszek M., Chabros L., Baczkowska T., Młynarczyk G. (2011). Strains of Klebsiella pneumoniae producing extended spectrum beta-lactamases, isolated from organ recipients. Transplant Proc., 43; 3128-3129.

[25] Paterson D.L., Hujer K.M., Hujer A.M., Yeiser B., Bonomo M.D., Rice L.B., Bonomo R.A., International Klebsiella Study Group (2003). Extended-spectrum $\beta$-lactamases in Klebsiella pneumoniae bloodstream isolates from seven countries: Dominance and widespread prevalence of SHV- and CTX-M-type $\beta$-lactamases. Antimicrob. Agents Chemother., 47: 3554-3560.

[26] Ferreira R.L., Da Silva B.C., Rezende G.S., Nakamura-Silva R., Pitondo-Silva A., Campanini E.B., Brito M.C., da Silva E.M., de Melo Freire C.C., da Cunha A.F., da Silva Pranchevicius M.C. (2019). High prevalence of multidrug-resistant Klebsiella pneumoniae harboring several virulence and $\beta$-lactamase encoding genes in a Brazilian intensive care unit. Front. Microbiol., 9: 3198.

[27] Brisse S., Fevre C., Passet V., Issenhuth-Jeanjean S., Tournebize R., Diancourt L., Grimont P. (2009). Virulent clones of Klebsiella pneumoniae: Identification and evolutionary scenario based on genomic and phenotypic characterization. PLoS One, 4: e4982.

[28] Lascols C., Peirano G., Hackel M., Laupland K.B., Pitout J.D. (2013). Surveillance and molecular epidemiology of Klebsiella pneumoniae isolates that produce carbapenemases: First report of OXA-48-like enzymes in North America. Antimicrob. Agents Chemother., 57: 130-136. 